\title{
Das Bielefelder Modell
}

\section{Wohnen im Quartier - mit Versorgungssicherheit, aber ohne Betreuungspauschale}

\author{
OLIVER KLINGELBERG \\ Oliver Klingelberg ist Soziologe und \\ war langjähriger Mitarbeiter der \\ Verbraucherzentrale Nordrhein- \\ Westfalen im Bereich "Wohnen \\ \& Pflege". Seit April 2011 ist er im \\ Sozialmanagement der Bielefelder \\ Gemeinnützigen Wohnungsgesell- \\ schaft tätig und dort $u$. a. verant- \\ wortlich für die Weiterentwicklung \\ von Quartierskonzepten. \\ o.klingelberg@bgw-bielefeld.de
}

\author{
In Bielefeld wird seit langem ein quartiersbezogener \\ Ansatz des Wohnens praktiziert. Einbezogen in \\ bestehende Wohnquartiere und in guter infrastruktureller \\ Anbindung bietet die örtliche Wohnungsbaugesellschaft \\ älteren Menschen oder Menschen mit Behinderung \\ komfortable und barrierefreie Wohnungen. Kombiniert \\ ist dieses Angebot mit einem Wohncafé und einem \\ Servicestützpunkt. Alle Mieter können auf die Hilfs- \\ und Betreuungsangebote zurückgreifen, müssen diese \\ aber nur im tatsächlichen Bedarfsfall bezahlen.
}

Das Thema Quartier hat Konjunktur. Mit dem Perspektivwechsel auf den Sozialraum werden neue, vielleicht passendere Antworten auf die hinlänglich bekannten sozialpolitischen Herausforderungen einer älter werdenden Gesellschaft erwartet. Dabei ist das Thema nicht wirklich neu.

Bereits Mitte der 1990er Jahre haben in Bielefeld die Bielefelder Gemeinnützige Wohnungsgesellschaft (BGW) und der Verein Alt und Jung e. V. mit dem »Bielefelder Modell« einen richtungsweisenden Quartiersansatz des selbstbestimmten Wohnens entwickelt, der bundesweit Aufmerksamkeit auf sich gezogen hat und mittlerweile auch in anderen Städten erfolgreich umgesetzt wird.

\section{Ausgangspunkte}

Die Stadt Bielefeld hat rund 325.000 Einwohner. Bereits heute ist schon mehr als jeder Fünfte über 65 Jahre alt. Dies liegt in etwa im bundesweiten Durchschnitt. Entsprechend hoch ist auch der Anteil der pflegebedürftigen Menschen, deren Zahl in einem Zeitraum von zehn Jahren um 20 Prozent gestiegen ist - ebenfalls vergleichbar mit vielen anderen Kommunen. Abweichend von der Norm wurde diese Zunahme an pflegebedürftigen Men- schen in Bielefeld jedoch ausschließlich im Bereich der häuslichen Versorgung aufgefangen.

Bereits 2007 hatte sich der Rat der Stadt fraktionsübergreifend dafür ausgesprochen, primär den Schwerpunkt in der Weiterentwicklung der Pflegeund Hilfestrukturen auf stadtteil- und wohngebietsnahe, ambulant betreute Wohnformen zu legen. Tatsächlich wurde seither der Anteil stationärer Pflegeplätze nur geringfügig ausgebaut.

Darin lag keineswegs eine fehlende Wertschätzung der stationären Pflege. Maßgeblich waren vielmehr die Einsicht, dass ein einseitiger Ausbau der stationären Pflege zur Deckung der Bedarfe nicht finanzierbar sei sowie die Wohnwünsche der Bürgerschaft, auch im Falle von Pflegebedürftigkeit im vertrauten Wohnumfeld verbleiben zu können.

Dieser Ratsbeschluss war jedoch nicht Beginn, sondern Meilenstein einer Entwicklung, die bereits zu Beginn der 1980er Jahren mit der Gründung zahlreicher ambulant betreuter Pflegewohngemeinschaften sowie selbstorganisierter Wohnprojekte in Bielefeld ihren Anfang genommen hatte. Standen dabei zunächst noch »Einzelprojekte « für eine enger gefasste Zielgruppe, nämlich Menschen mit Behinderung, chronisch kranke und pflegebedürftige Menschen, 


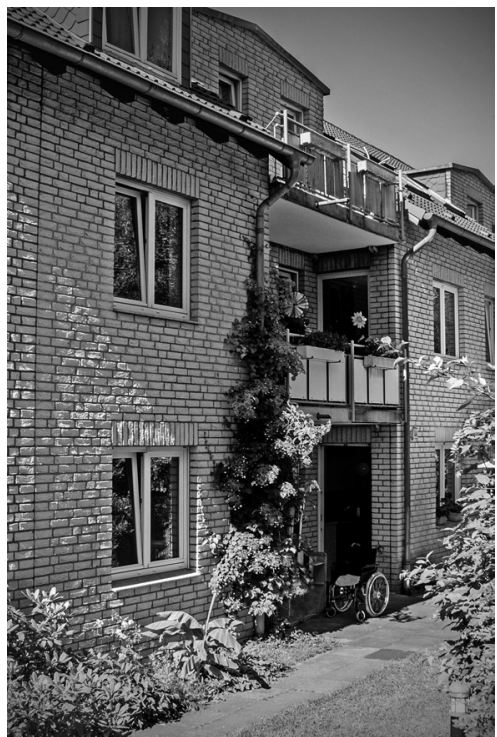

Im ersten Wohnprojekt in der Dahlemer Straße waren bereits alle vier Bausteine des Bielefelder Modells verwirklicht: Quartiersbezug, Barrierefreiheit, Rund-um-die-Uhr-Präsenz eines sozialen Dienstleisters, Aufbau und Aktivierung einer tragenden sozialen Infrastruktur.

im Vordergrund, rückte nach und nach das gesamte Quartier als "Sozialraum « in den Fokus. Es entstand die Vision, eine umfassende, möglichst 24-stündige Versorgungssicherheit ohne Grundservicepauschalen als wohnbegleitende Dienstleistung in bestehende Wohnviertel einzubetten. Oder in Anlehnung an Klaus Dörner besser formuliert: Die Hilfe sollte zu den Menschen, nicht die Menschen zu den Hilfestrukturen gebracht werden. (1)

Ausgehend von diesem Denkansatz entwickelten Anfang der 1990er Jahre die Bielefelder Gemeinnützige Wohnungsgesellschaft und der Verein Alt und Jung e. V. jenes Konzept, das als »Bielefelder Modell« bundesweit bekannt und mittlerweile zu einem Synonym für ein quartiersbezogenes selbstbestimmtes Wohnen mit Versorgungssicherheit geworden ist.

\section{Die vier Bausteine des Modells}

Die Bielefelder Gemeinnützige Wohnungsgesellschaft, 1950 als mehrheitlich kommunales Wohnungsunternehmen gegründet und zum größten Immobiliendienstleister der Region gewachsen, und der 1978 entstandene Verein Alt und Jung hatten bereits zuvor bei einer
Reihe von ambulant betreuten Wohnprojekten kooperiert. In enger Abstimmung mit der Stadt Bielefeld entstand dann als gemeinsames Pilotprojekt ein Neubau mit 55 barrierefreien Wohnungen an der Dahlemer Straße, der 1996 bezogen werden konnte. Hier finden sich erstmals alle vier konzeptionellen Bausteine des Bielefelder Modells:

- Der Quartiersbezug: Statt einer »Versorgungsinsel « rückt ein vorhandenes Wohnquartier in seiner gesamten Bewohnervielfalt in den Blickpunkt. Die Versorgung ist dabei auf eine überschaubare Nachbarschaft in einem Radius von etwa tausend Metern oder etwa 800 bis 1.000 Haushalte begrenzt, sodass alle Bewohner eines Quartieres bei Bedarf durch den sozialen Dienstleister innerhalb weniger Minuten fußläufig oder mit dem Fahrrad erreicht werden können.

- Barrierefreiheit: Ausgangspunkt aller Überlegungen ist der Mensch mit dem höchsten Hilfebedarf. Um auch für diesen ein möglichst selbstbestimmtes Leben in einer eigenen Wohnung ermöglichen zu können, ist Barrierefreiheit ein elementares Kriterium. Das heißt allerdings nicht, dass alle Wohnungen eines Quartiers barrierefrei sein müssen. Obwohl Barrierefreiheit für alle einen Komfortstandard darstellt, ist sie nur für eine Teilmenge der Bewohner im Quartier ein Muss. Für diese Personen bedarf es allerdings einer Mindestzahl von am Anfang der Projekte. Allerdings gilt auch hier, keine Regel ohne Ausnahme. Mittlerweile finden sich dort, wo es keine geeigneten Grundstücke für einen Neu- oder Ersatzbau gab, auch gelungene Umsetzungen in Bestandsimmobilien, insbesondere dann, wenn diese Gebäude bereits über eine schwellenfreie Erschließung durch eine Aufzugsanlage verfügten. Schwierig bleiben die Wohnungsgrundrisse, die nachträglich selten in allen Teilbereichen der DIN entsprechend umgestaltet werden können, sodass hier besser von einem »barrierearmen" statt barrierefreien Baustandard zu sprechen ist.

- 24-stündige Präsenz eines sozialen Dienstleisters: In diesem Um- oder Neubau finden sich auch die Büros und Funktionsräume des sozialen Dienstleisters, denn neben der Barrierefreiheit stellt die 24-stündige Präsenz eines kompetenten sozialen Dienstleisters die zweite Säule einer umfassenden Versorgungssicherheit im Quartier dar. Personen mit einem intensiven, gegebenenfalls auch nächtlichen Pflegebedarf können so engmaschig begleitet werden. Über das klassische Hausnotrufsystem kann gewährleistet werden, dass jeder, der dies in der Nachbarschaft wünscht, binnen weniger Minuten Hilfeleistungen anfordern kann. Die alleinige Präsenz des sozialen Dienstleisters im Quartier ist dabei nicht mit Kosten verbunden. Es werden nur Kosten für Dienstleistun-
"Zentrale Elemente des Konzeptes sind ein Mix aus baulicher Infrastruktur und sozialer Dienstleistung sowie aus professionellen und informellen Netzwerken« barrierefreien Wohnungen möglichst gemäß der DIN 18025 bzw. jetzt DIN 18040. Da diese Wohnungen in aller Regel in den vorhandenen Quartiersstrukturen fehlen und oftmals weder wirtschaftlich noch bautechnisch im Rahmen einer strukturellen Wohnungsanpassung im Bestand realisierbar sind, steht somit - wie in der Dahlemer Straße - meist ein Neubau gen abgerechnet, die tatsächlich in Anspruch genommen werden, also beispielsweise die Bereitstellung des Hausnotrufes oder konkrete hauswirtschaftliche oder pflegerische Hilfen. Es gibt also keine Serviceoder Betreuungspauschalen. Die Entgelte entsprechen den üblichen Abrechnungssätzen der ambulanten Pflege. 


\section{Zuhause im Quartier}

Der Paritätische Wohlfahrtsverband in Nordrhein-Westfalen setzt innovative Wohnmodelle für alle Generationen um. Denn zu Hause alt werden, das wünschen sich rund zwei Drittel der Seniorinnen und Senioren in Deutschland. Die Pflegestatistik zeigt, dass bundesweit 70 Prozent der pflegebedürftigen Menschen zu Hause versorgt werden - sie wollen im Alter in ihrer vertrauten Umgebung wohnen bleiben. Aber auch die jüngere Generation und Familien mit Kindern möchten sich in ihrem Stadtviertel wohl fühlen, soziale und nachbarschaftliche Netzwerke nutzen.

Bereits 2006 haben sich der Paritätische Nordrhein-Westfalen, der VdW Rheinland Westfalen und der Deutsche Mieterbund Nordrhein-Westfalen in der "Dortmunder Erklärung" (www.wohnen-im-alternrw.de) verpflichtet, nutzerfreundliche Wohnmodelle für ältere Menschen zu entwickeln und nachhaltig zu gestalten. Gemeinsam mit seinen Mitgliedsorganisationen und Kooperationspartnern setzt sich der Verband für eine quartiersnahe Versorgung ein. Generationsübergreifendes Wohnen im angestammten Quartier ist der Leitgedanke für diese innovativen Wohnformen. Unter dem Dach des Paritätischen versammeln sich derzeit rund 70 ambulant betreute Wohngemeinschaften sowie knapp 20 generationsübergreifende Wohn- und zahlreiche Quartiersprojekte.

Wenn ältere Menschen aufgrund einer Erkrankung oder Behinderung den Alltag nicht mehr alleine bewältigen können, benötigen sie niedrigschwellige Hilfsangebote innerhalb ihres Wohnviertels. Nachbarschaftliche Netzwerke, eine Haushaltshilfe und ambulante Pflegedienste unterstützen die Betroffenen, damit sie ein selbstständiges Leben führen können. Auch eine Wohnraumberatung hilft ihnen, ihre Wohnung barrierearm oder barrierefrei zu gestalten. Ist der

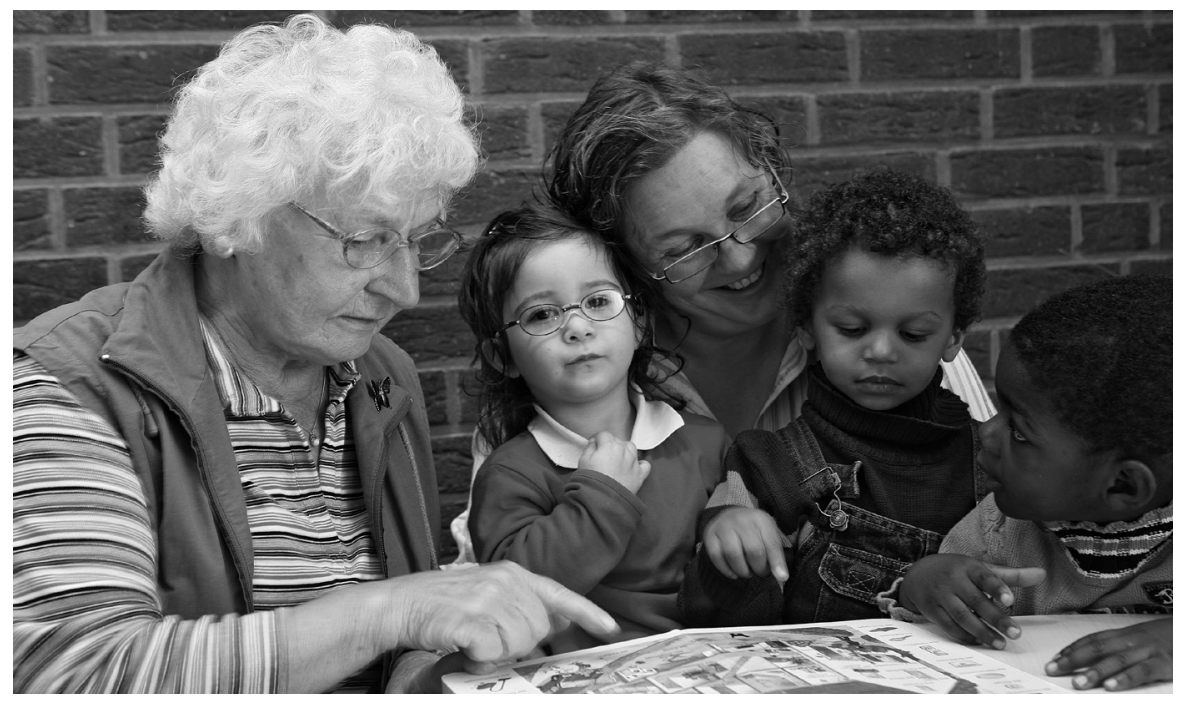

Verbleib in der eigenen Häuslichkeit nicht mehr möglich, kann der Umzug in eine ambulant betreute Wohngemeinschaft eine Alternative sein. Diese Versorgungsart lässt sich aufgrund ihrer überschaubaren Größe sehr gut ins Quartier integrieren und bietet den BewohnerInnen viele Vorteile - unter anderem werden sie dort rund um die Uhr betreut.

Generationsübergreifende Wohnprojekte, von denen es in Nordrhein-Westfalen noch deutlich zu wenige gibt, zeigen, wie man ein Quartier alters- und sozialdurchmischt entwickelt. Um ein Stadtviertel lebendig zu gestalten, müssen sich ältere und jüngere Menschen, Familien und Alleinerziehende mit Kindern dort wohl fühlen. Alle Generationen wünschen sich ein Umfeld, das geprägt ist von Aspekten wie Geborgenheit, Sicherheit, Vertrautheit, und Erreichbarkeit - ihr Zusammenleben erfordert jedoch auch Toleranz und Kompromissbereitschaft. Darüber hinaus sind Nachbarschaftsvereine ein wichtiges Bindeglied, um Kontakte innerhalb der Nachbarschaft zu pflegen: Sie schaffen Orte der
Begegnung und des Engagements mitten im Quartier.

Der Paritätische Wohlfahrtsverband verstärkt mit seinem Kompetenzzentrum "Wohnen im Alter NRW" und dem gleichnamigen Informationsportal im Internet seine Aktivitäten zur Quartiersentwicklung. In den vergangenen Jahren hat der Verband einen Zulauf an gemeinnützigen Vereinen verzeichnet, die im breiten Themenfeld "Wohnen im Alter" ihre Vorstellungen verwirklichen möchten. Dabei wird der Paritätische seine Mitglieder unterstützen und helfen, fehlende Angebote zu initiieren sowie Hindernisse aus dem Weg zu räumen. Auch die Landesregierung fördert strukturell und ressortübergreifend mit dem "Masterplan Quartier" die Umsetzung von Quartiersprojekten.

Franz Schumacher

Fachreferent "Wohnen im Alter" und "Ambulante Pflege « beim Paritätischen Wohlfahrtsverband, Landesverband NordrheinWestfalen e. V.

www.wohnen-im-alter-nrw.de
- Aufbau und Aktivierung einer tragenden sozialen Infrastruktur: So wie es keine vollständige Barrierefreiheit geben kann und muss, ist auch die umfassende Versorgungssicherheit innerhalb des Quartiers allein durch hauptamtliche Strukturen kaum möglich und auch nicht erstrebenswert. Vielmehr gilt es, die vorhandenen Selbsthilfepotenziale der Menschen zu stärken, informelle Netzwerke aufzubauen und $z u$ erhalten, das Ehrenamt und bürgerschaftliches Engagement zu fördern. Damit ist die dritte zentrale Säule im
Bielefelder Modell angesprochen: die aktive Nachbarschaft, in der sich die Menschen entsprechend ihrer Fähigkeiten und Bedarfe füreinander einbringen.

Um dieses Ziel zu erreichen, bedarf es zunächst im engeren Bedeutungssinne des passenden Raumes. Daher ist wesentlicher Teil der Konzeptidee die Erstellung eines »Wohncafés", als Ort für ein privates Angebot von Bürgern für Bürger in der Nachbarschaft. Dieses wird seitens der Bielefelder Gemeinnützigen Wohnungsgesellschaft kostenfrei zur Verfügung gestellt und ist offen für alle Bürger im Quartier, unabhängig von Alter, Kultur oder Lebenslage. Insbesondere schafft es aber ein Angebot für Menschen, die aufgrund fehlender familiärer Netzwerke oder durch Krankheit oder Pflegebedürftigkeit einen besonderen Teilhabe- und Fürsorgebedarf haben. Tagesstrukturierende Ankerpunkte stellen die täglichen Mahlzeiten dar, die durch ehrenamtliche Köche zum Selbstkostenpreis zubereitet werden. Darüber hinaus bietet das Wohncafé Raum für weitere Aktivitäten, wie ge- 
meinsames Spielen, Kultur-, Lern- und Sportangebote.

Ebenso wie das Wohnungsunternehmen ist auch der soziale Dienstleister nur Gast im Wohncafé und ausdrücklich nicht »Träger « oder »Betreiber « der Gemeinschaftsräume. Seine Funktion beschränkt sich auf den Aufbau, die Koordinierung und nachhaltige Aktivierung bürgerschaftlichen Engagements sowie die fachliche Begleitung und Qualifizierung der ehrenamtlich Tätigen.

Damit sind die zentralen Elemente des Konzeptes als Mix aus baulicher Infrastruktur und sozialer Dienstleistung sowie aus professionellen und informellen Netzwerken bereits benannt.

\section{Versorgungssicherheit ohne Betreuungspauschale: So geht es}

Was zunächst ebenso simpel wie überzeugend klingt, muss jedoch in der Praxis für alle Beteiligten - Wohnungsunternehmen, sozialer Dienstleister und Kunde - auch wirtschaftlich umsetzbar sein.

Um niemanden, der eine barrierefreie Wohnung benötigt, allein schon aufgrund seiner Einkommenslage auszuschließen, findet sich bei den neu erstellten oder im Bestand umgebauten Wohnungen immer eine Mischung aus frei finanzierten und öffentlich geförderten Wohnungen. Letztere sind in Wohnungsgröße und Mietzins auch für Personen geeignet, die Grundsicherung oder andere Transferleistungen beziehen. (2)

Der Kooperationspartner erhält ein Vorschlagsrecht bei der Belegung von vier bis sechs der barrierefreien Wohnungen. Damit ist von Beginn an gewährleistet, dass immer Mieter im Haus wohnen, die in einem umfänglichen Maße bereits Dienstleistungen benötigen und nachfragen. Der Anteil der Menschen mit Hilfe- und Pflegebedarf im Hause sollte dabei jedoch insgesamt ein Drittel nicht überschreiten, damit auch innerhalb der Hausgemeinschaft das Wohnen und nicht die Pflege im Vordergrund steht. Auch hier soll innerhalb der Nachbarschaft wechselseitige Hilfestellung möglich bleiben und keine »Pflegeinsel « im Quartier entstehen.

Dieses Vorschlagsrecht bei der Belegung von Wohnungen gibt dem Koope- rationspartner eine Basis, die erforderliche 24-Stunden-Präsenz im Quartier sicherzustellen. Als ergänzender Baustein finden sich in allen Projekten ein bis zwei barrierefreie »Gästewohnungen «. Diese werden vorrangig im Kontext der ambulanten Verhinderungspflege gemäß $\ 39$ SGB XI genutzt oder stehen Personen zur Verfügung, die aufgrund ihrer gesundheitlichen Situation vorübergehend nicht in der Lage sind, einen eigenen Haushalt zu führen. In aller Regel erfolgt auch hier eine Begleitung durch den Kooperationspartner im Quartier. (3)

Allerdings reichen diese Steuerungsmodule noch nicht aus, die erforderliche durchgängige Versorgungssicherheit im Quartier durch einen sozialen Dienstleister auf ein solides wirtschaftliches Fundament zu stellen und den Verzicht auf eine Betreuungspauschale zur Refinanzierung der Präsenzzeiten im Quartier aufzufangen. Hierfür ist eine aktive Quartiersarbeit zwingend erforderlich, um weitere Kunden im näheren Wohnumfeld zu akquirieren und den Standortvorteil der niederschwelligen Präsenz durch Servicestützpunkt und Aktivitäten im Wohncafé auszunutzen.

Für diesen Perspektivwechsel von der Wohnung zum Sozialraum bedarf es einer ergänzenden Sozialarbeit. Diese kann in den Anfängen eines Projektes durch Fördermittel finanziell aufgefangen werden. (4)

Insgesamt gilt also für den ambulanten Dienst als Kooperationspartner, dass ein Umdenken und anderes Selbstverständnis zugrunde gelegt wird. Er darf sich nicht allein als Pflegedienst im Versorgungsbereich der Sozialgesetzbücher V und XI definieren, sondern muss in einem sehr viel umfassenderen Sinne als sozialer Dienstleister im Quartier agieren, mit den entsprechenden Qualifikationen und Leistungsvereinbarungen, beispielsweise in den Bereichen der Eingliederungshilfe, der psychiatrischen Pflege oder auch in der Kinderund Jugendhilfe.

\section{Fazit}

Dass neues Wohnen funktionieren kann, dokumentieren allein 14 Projekte der Bielefelder Gemeinnützigen Wohnungsgesellschaft, die mit unterschiedlichen Projektpartnern im Bielefelder Stadtgebiet umgesetzt worden sind. (5)
Maßgeblich hierfür ist sicherlich auch, dass alle Projektbeteiligten dabei in einem engen und kontinuierlichem Austausch stehen, die Stadt Bielefeld und insbesondere die Bürger der jeweiligen Quartiere mit inbegriffen.

Transparenz, Akzeptanz und aktive Beteiligung sind die Voraussetzungen einer stetigen konzeptionellen Weiterentwicklung, von der Kommune, Wohnungsunternehmen, sozialer Dienstleister und letztendlich und vor allem wir alle als Bürger profitieren.

\section{Anmerkungen}

(1) Vgl. Klaus Dörner: Leben und Sterben, wo ich hingehöre. Dritter Sozialraum und neues Hilfesystem. Neumünster 2007.

(2) Der Grenzwert der angemessenen Kosten der Unterkunft liegt derzeit in Bielefeld bei 4,64 Euro je Quadratmeter. Eine Besonderheit ist, dass seit 2007 dieser Grenzwert um einen Klimabonus nach energetischen Gebäudekriterien in drei Stufen bis auf 5,29 Euro je Quadratmeter erhöht werden kann. Das durchschnittliche Mietpreisniveau stellt sich aktuell in Bielefeld mit 5,81 Euro je Quadratmeter dar (vgl. hierzu: Stadt Bielefeld (Hg.): Wohnungsmarktbericht 2012). Der Mietzins der frei finanzierten Wohnungen der Bielefelder Gemeinnützigen Wohnungsgesellschaft im Bielefelder Modell beträgt 8,50 Euro je Quadratmeter.

(3) Nur der Form halber sei erwähnt, dass selbstverständlich Wohnen und Dienstleistung konsequent vertraglich voneinander getrennt sind und bei der Wahl eines Anbieters sowohl in den barrierefreien Wohnungen als auch im gesamten Quartier absolute Wahlfreiheit gewährleistet ist.

(4) Das Deutsche Hilfswerk (DHW) fördert beispielsweise Personalund Sachkosten der Quartiersentwicklung für einen Projektzeitraum von maximal drei Jahren mit bis zu 100.000 Euro.

(5) Neben dem Verein Alt und Jung e. V. wurden Projekte mit dem Hauspflegeverein, der Arbeiterwohlfahrt und dem Deutschen Roten Kreuz umgesetzt. 